\title{
Reaction Condition Dependency of Propagating Behavior in the Polymerization Reaction by Thermal Front
}

\author{
Do Sung Huh," Sang Joon Choe, and Burm-Jong Lee \\ Department of Chemistr, Inje Lniversity, Kimhae 621-749, Korea \\ Received July 10,2001
}

\begin{abstract}
In this study. the dependency of the behavior of propagating front on the reaction condition in frontal polymerization reaction has been studied. We have used some multifunctional acrylates as a monomer and ammonium persulfate as an initiator for the polymerization reactions. In frontal polymerization. a method of producing polymeric materials via a thermal front that propagates through the unreacted monomer/initiator solution the behavior of self propagating front shows various dynamic patterns depending on the reaction condition. We have obtained some spin modes of propagating front in the number of "hot spots" or "spin heads" by changing the reaction condition. The effect of the reactor tube diameter on the mode of propagating front has also been studied by using some reactor tubes with different size of tube diameter and it has been examined in some detail by adopting an experimental method of two-tubes system.
\end{abstract}

Keywords : Propagating front. Frontal polymerization. Spin modes.

\section{Introduction}

Thernal autocatalysis can generate propagating fronts, as seen in combustion. The same effect occurs in exothernic polymerization reactions. such as free-radical polymerization and epoxy curing. Frontal polymerization is a mode of converting monomer into polymer via a localized reaction zone that propagates as a front. most often through the coupling of thermal diffusion and Arrhenius reaction kinetics.

Alexander $\mathrm{G}$. Merzhanov and his colleagues discovered the process of Self-Propagating High-temperature Synthesis (SHS) in 1967 to prepare technologically useful ceramics and internetallic compounds. ${ }^{1.2}$ A compressed pellet of reactants was ignited at one end that resulted in a self-propagating combustion wave. The method has the advantages that the initial stimulus is the only energy input required and that superior materials are produced. Therefore the polvmerization method has benefits over traditional methods of polymerization process in point of reduced energy costs, reduced waste production and unique morphology. And it has been demonstrated as a method for functionally gradient polymeric materials ${ }^{3}$ and may have utility in preparing large composites. ${ }^{+}$ A desirable feature of frontal polymerization is rapid and uniform conversion of monomer to polymer. Also. the absence of solvent eliminates the need to separate the polymer from the solvent and residual monomer, which requires energy and can have environmental ranifications.

A rich variety of dynanical behavior has been observed in the SHS systems. including planar fronts. spin modes, ${ }^{5.6}$ and chaotic reaction waves. ${ }^{7}$ Dynamics have also been studied numerically and analytically. ${ }^{8}$ Frontal polymerization is an organic and more amenable analog of self-propagating hightemperature synthesis (SHS) of inorganic compounds. Due to lower temperatures involved in the process and to slower velocities, frontal polymerization systems are easier to handle than SHS systems for studying the behavior of propagating fronts. The work relating with the frontal polymerization up to 1984 was reviewed by Davtyan $e t a l .{ }^{9}$ and Pojman $e t a l$. provided an update in 1996. ${ }^{10}$

An experimental setup and detailed experimental procedure for the frontal polymerization in acrylate monomer system has been described by Huh $e t$ al. recently. ${ }^{11}$ In the study Huh et al. have introduced some stable propagating front in the acrylate monomer system and also the possibility of an unstable mode in frontal polymerization by small variation of the reaction condition.

Along with empirical studies of frontal polymerization systems, different front dynamics were also theoretically investigated." Of particular interest to the dynamics of propagating front is the spin-mode characterized by a nonplanar front with one or more high-temperature regions, "hot spots". that move in a helical path along the axis of the reaction vessel. The first true spin mode for a system with a constant front velocity was reported by Pojman et al. in the methacry lic polymerization. ${ }^{13}$ In the system. the frontal polymerization exhibited spin modes when the initial temperature was lowered to $0^{\circ} \mathrm{C}$. Spin modes at room temperature were first observed by Masere and Pojman in the frontal polymerization of a diacrylate monomer. ${ }^{14}$ The number of hot spots was affected by the front temperature which was controlled by an inert diluent. and by the degree of crosslinking which was controlled by varying the ratio of the monoacry late monomer to the multifunctional acrylate monomer.

In this study, the dependency of the behavior of propagating front on the reaction condition in frontal polymerization has been studied by adopting various reaction conditions. The effect of the used monomer on the behavior of propagating front has been studied by comparing between multifunctional acrylate monomer systems. Some diacrylate and triacrylate monomers were used for this study. We have obtained some spin modes of propagating front in the number of "hot spots" or "spin heads" in some reaction conditions. And two 
diacrylate monomers of 1,6-hexanedioldiacrylate and 1,6hexanediol dimethacrylate which have a similar structure have been used in order to study the effect of a small difference of molecular structure in the used monomer on the behavior of propagating front

The effect of the reactor tube diameter has also been examined by using some reactor tubes with different size of tube diameter and it has been studied in some detail by adopting an experimental method of two-tubes system.

\section{Experimental Section}

Reagents. 1.6-hexanedioldiacrylate (HDDA). 1,6-hexanediol dimethacrylate (HDDMA), Triethyleneglycoldimethacry late (TGDMA), trimethylolpropane triacry late (TMPTA) were used as monomers with anmonium persulfate initiator for the study. Bromophenol Blue (BPB) was used as an indicating dye to visualize the propagating front more clearly. The dye dissolved in dimethyl sulfoxide (DMSO) was used. Ammonium persulfate had poor solubility in monomer but the addition of DMSO improved the initiator solubility. All chemicals were purchased from Aldrich and used as received without further purification

Procedures. The initiator solution was prepared by dissolving amonium persulfate into $15 \mathrm{~mL}$ DMSO diluent. The quantity of dissolved ammonium persulfate was changed by the volume of the used monomer in each experiment. and its ratio was about $1 \%$ to the used monomer by mole percent. To monitor the propagation of front by the color change from unreacted monomer solution to reacted polymer solid. $0.04 \mathrm{M}$ Bromophenol Blue solution in DMSO was used. A reaction mixture was prepared by adding some volume of monomer which was varying in the ratio of monomer to DMSO diluent in each experiment into the $15 \mathrm{~mL}$ initiator solution in the $50 \mathrm{~mL}$ beaker followed by addition of 0.15 $\mathrm{mL}$ of the Bromophenol Blue solution to give a green color. This solution was charged into a capped test tube, and frontal polymerization was initiated with a soldering iron. Video images of the propagating front were obtained using a digital camcorder (Handycam video Hi8. Sony TRV 320) and were digitized on $\mathrm{PC}$ computer using FireBird DV.

\section{Results and Discussions}

The effect of used monomer and reaction condition on the hehavior of propagating front. We have obtained some experimental results that the dynamic behavior of propagating front is largely dependent on the used monomer in frontal polymerization process. A stable planar progress of propagating front has been obtained mostly in diacrylate monomer system. In this study HDDA was used primarily as a diacrylate monomer. A typical image of the polymerization process by a stable propagating front has been introduced in Figure 1 . The dark green reactant solution turned pale yellow as the front progressed downwards with polymerization reaction. The color change in the front is due to the conversion of Bromophenol Blue to its another form via free radical

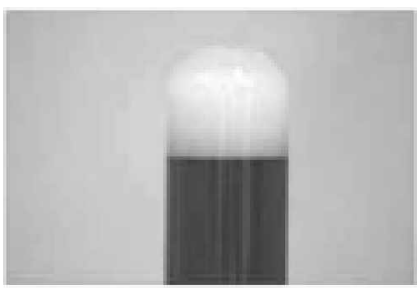

$30 \mathrm{~s}$

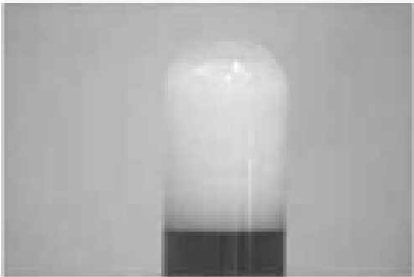

905

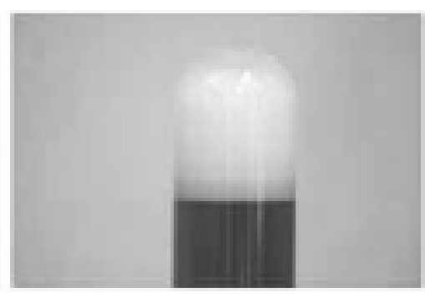

$60 \mathrm{~s}$

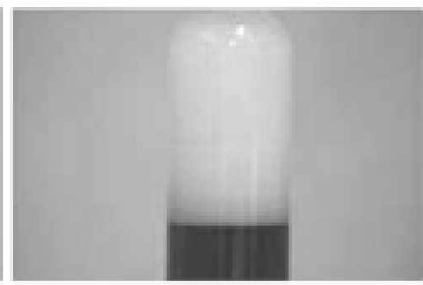

$120 \mathrm{~s}$

Figure 1. A typical image of a stable propagating front in the HDDA trontal polymerization. The dark green zone shows the tresh reactant solution and the pale yellow zone is the polymer synthesized.

coupling. However. the indicating dye is more useful in the polymerization process in which the propagating front is progressing with a nonplanar mode or a spin mode. In the case, the nonplanar or periodic spin mode could be visualized more clearly in the number of hot spot and in the direction of spimining front.

A typical image of propagating front with a nonplanar mode in frontal polymerization reaction is introduced in Figure 2. It shows a single-head spin mode with discrete time. The used monomer is TMPTA which has the molecular structure of a triacrylate. Figure 2 shows that one bright hot spot is leading the propagating front by moving with a helical path along the cylinder tube and leaving a bright track as it moves in a single-head spin mode. In HDDA monomer system a similar single-head spin mode

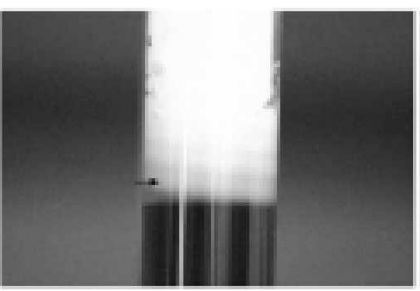

$200 \mathrm{~s}$

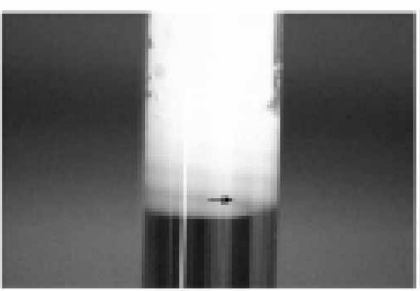

$210 \mathrm{~s}$

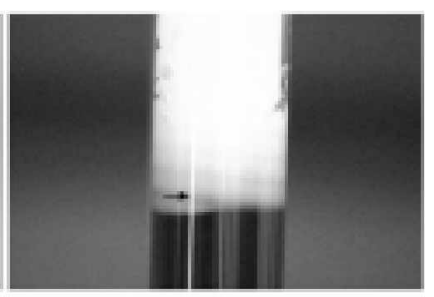

$205 s$

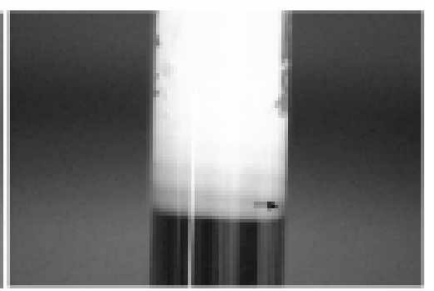

$215 \mathrm{~s}$

Figure 2. An image showing a single-head spin mode in the TMPTA frontal polymerization. In this case, the reactant mixture was prepared by mixing $28 \%$ of monomer in DMSO diluent. The arrow indicates the direction of moving front head. 


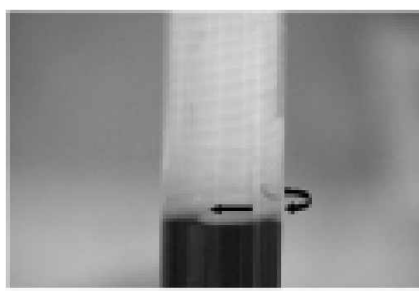

$250 \mathrm{~s}$

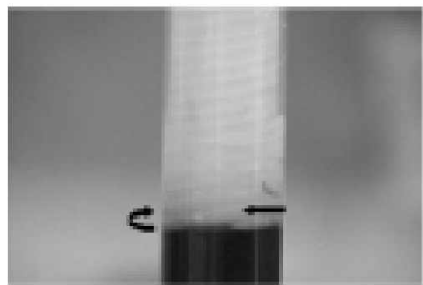

$260 \mathrm{~s}$

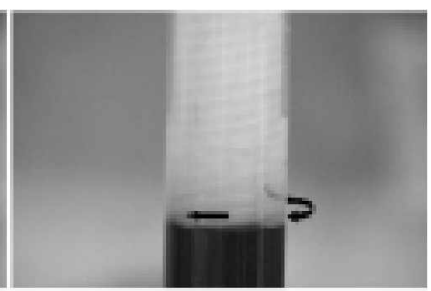

$255 \mathrm{~s}$

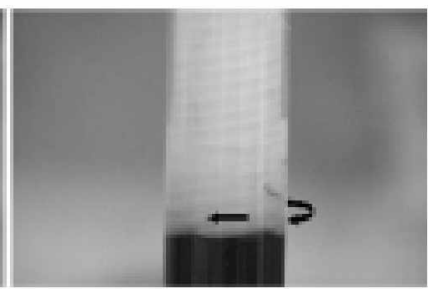

$265 \mathrm{~s}$

Figure 3. An image showing a two-heads spin mode in the TMPTA trontal polymerization by small increasing the ratio of monomer composition in DMSO diluent. Two arrows indicates the two heads moving with same direction.

has also been obtained in some reaction condition. but the single-head spin mode in HDDA monomer system was not so prone to be obtained comparing to the TMPTA monomer system in same reaction condition.

Figure 3 shows a typical image of propagating front with a two-heads mode in TMPTA system by a small change of the chemical reaction condition. In the system the monomer concentration was increased a little. In the two-heads spin mode two bright hot spots are leading the propagating front by moving with a helical path along the cylinder tube. The two spots indicated by two arrows in Figure 3 are moving with same direction in the propagating front. It was not easy to capture the two spots at same time in a fixed position since one spot appearing just as its predecessor is disappearing from the view. In the reaction system using diacrylate monomer of HDDA, the two-heads spin mode was obtained rarely. The obtained polymer rod have a spiral trace when the polymerization reaction was done by the propagating front with a single-head spin mode or with a two-heads spin mode.

When the monomer concentration was further increased, a flat-like or rippled front ${ }^{1 \mathrm{l}}$ was obtained in both HDDA and TMPTA monomer system. However, in some reactions using TMPTA monomer, a zig-zag mode was obtained. In the case. two hot spots move from the opposite direction and collide each other as shown in Figure 4 . The pattern of propagating front is similar with the two-heads mode in the number of moving hot spots but the moving direction of the two spots is different from the two-heads mode. The opposite direction in a zig-zag pattern is well explained by the moving arrows of Figure 4 . And also the visual analysis of the polymer rod obtained from the zig-zag mode shows a different pattern in the spiral trace when it is compared with a polymer rod obtained by a single-head or two-heads spin mode. The spiral trace in the polymer rod obtained from the zig-zag mode was not well defined and it showed an irregular and a complex pattern. The zig-zag pattern was not

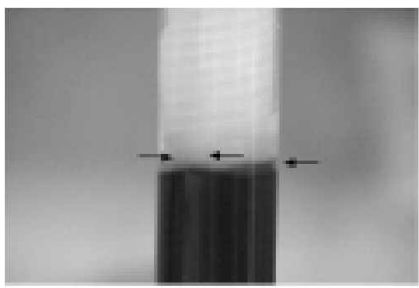

$250 \mathrm{~s}$

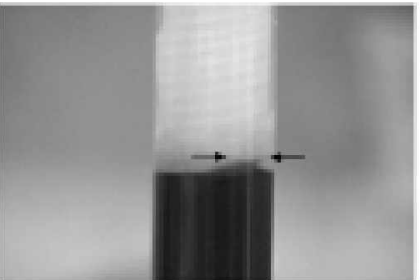

$254 \mathrm{~s}$

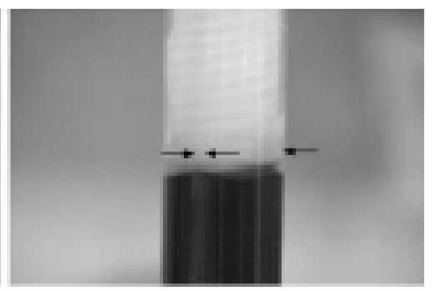

$252 \mathrm{~s}$

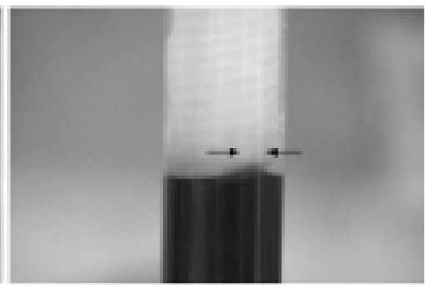

$256 s$
Figure 4. An inage showing a zig-zag mode in the TMPTA frontal polymerization. In the mode, two heads leading the thermal front for frontal polymerization are moving with different direction and collide each other as shown in the arrows.

obtained in HDDA monomer system in any reaction condition of this study. This result can be interpreted by a difference of the degree of crosslinking in the frontal polymerization between diacrylate and triacrylate monomer system. The bifurcation number determining the stability of a thermal front is the Zeldovich number., ${ }^{13}$

$$
Z=\left(T_{n i}-T_{0}\right) / T_{n i} \times E_{\text {effi }} / R T_{m}
$$

By the equation frontal polymerization reaction is assumed to be occurred in an infinitely narrow region in a single step with activation energy $E_{0 f}$. initial temperature $T_{0}$ and maximum temperature $T_{m}$. By theoretical analysis. ${ }^{13}$ the planar mode is stable if $Z<Z_{i r}=8.4$ and unstable if $Z>Z_{c r}$. By varying the Zeldovich number up to the stability threshold. subsequent bifurcations leading to higher spin mode instabilities can be observed. The used triacrylate monomer has a higher value of $E_{\text {eff }}$ than that of diacrylate monomers because of crosslinking structure in the polymerization process. Thus. the reaction system of triacrylate monomer could have a higher $Z$ value than that of monoacrylate or diacrylate monomer syystem. Thus. the zig-zag pattern was not obtained in HDDA monomer system while it could be obtained in TMPTA monomer system in this study.

The effect of reaction condition and used monomer to the propagating front has been summarized in Table 1. The velocity of propagating front shown in Table 1 has been obtained by plotting the front position with progressing time. The plot has produced a straight line whose slope is the front velocity. The obtained velocity of propagating front in the HDDA and TMPTA polymerization was about $1.0 \mathrm{~cm} / \mathrm{min}$.

We can see an interesting experimental result in Table 1 in relation to the behavior of frontal polymerization of some monomers. Table 1 shows that it is impossible to obtain propagating front in HDDMA monomer system although the frontal polymerization of HDDA has been obtained in same reaction condition. There is only a small difference 
Table 1. The effect of used monomer on the behavior of propagating front in frontal polymerization. HDDA, HDDMA, and TGDMA are diacrylate monomers, and TMPTA is triacrylate monomer. And the difference between HDDA and HDDMA is in the presence of methyl functional group in the acrylic double bond

\begin{tabular}{cccc}
\hline & $\begin{array}{c}\text { Volume percentage } \\
\text { of monomer in } \\
\text { DMSO diluent }(\%)\end{array}$ & Front behavior & $\begin{array}{c}\text { Front velocity } \\
\text { (cnn/min) }\end{array}$ \\
\hline HDDMA & 31 & no propagation & 0.0 \\
TGDMA & 40 & no propagation & 0.0 \\
& 31 & slow propagation & 0.2 \\
HDDA & 40 & flat propagation & 0.4 \\
& 28 & single-head mode & 0.5 \\
& 31 & two-heads mode & 0.7 \\
TMPTA & 36 & flat propagation & 1.2 \\
& 32 & single-head mode & 0.6 \\
& 35 & two-heads mode & 1.0 \\
& 40 & zig-zag mode & 1.2 \\
& tlat or rippled front & 2.0 \\
\hline
\end{tabular}

between HDDA and HDDMA in the molecular structure. ${ }^{15}$ The difference is in that HDDMA has a methyl functional group instead of hydrogen in the acry lic double bond. In the frontal polymerization of this study using HDDMA monomer, the initial front formed by soldering iron on the top of the capped tube did not progress and stopped some times after regardless of HDDMA volume percentage in the DMSO diluent. The reason can be explained by considering the principal steps of a free-radical polymerization process.

Most free-radical polymerization reactions are highly exothermic and able to support frontal polymerization regime. A free-radical polymerization with a thermal initiator can be approximately represented by a three-step mechanism. ${ }^{15}$ First. an unstable initiator decomposes to produce radicals:

$$
\mathrm{I} f \rightarrow 2 \mathrm{R} \text {. }
$$

where $f$ is the efficiency: which depends on the initiator type and the solvent. A radical can then add to a monomer to initiate a growing poly'mer chain:

$$
\begin{gathered}
\mathrm{R} \cdot+\mathrm{M} \rightarrow \mathrm{P}_{1} . \\
\mathrm{P}_{12} \cdot+\mathrm{M} \rightarrow \mathrm{P}_{\mathrm{n}-1} .
\end{gathered}
$$

The propagation step $(\mathrm{R} 3)$ continues until a chain terminates by reacting with another chain:

$$
\mathrm{P}_{\mathrm{n}} \cdot+\mathrm{P}_{\mathrm{m}} \rightarrow \mathrm{P}_{\mathrm{n}-\mathrm{n}}
$$

The major heat release in the polymerization reaction occurs in the propagation step. The thermal autocatalysis for the frontal polymerization takes place in the initiator decomposition step because the initiator radical concentration is the main control for the total polymerization rate. Therefore the experimental result that frontal polymerization does not progress when HDDMA is used as a monomer means that there are some kinetic and thermodynamic difference between HDDA and HDDMA monomer in the steps represented by (Rl)-(R4). When HDDMA has been compared to HDDA. the structure of HDDMA could have a higher steric hinderence and a larger electron donation effect by the methyl functional group in the acrylic double bond. The difference is able to cause a lower exothermic reaction and a slower propagation rate in the HDDMA polymerization reaction. This interpretation is supported by an another experiment using TGDMA monomer system as shown in Table 1. TGDMA is also a kind of diacrylate monomer which has methacrylate structure. The velocity of the propagating front of TGDMA system was much slower than that of HDDA system in the same reaction condition. However, we could not analyze the difference of the behavior of propagating front between dimethacrylate and acrylate monomer system quantitatively by this study alone. It should be studied continuously by repeated experiments using various acrylate monomers which have similar molecular structure.

The effect of tube diameter on the behavior of propagating front. For a cylindrical reactor geometry the number of spin heads or hot spots is also dependent on the diameter of the reactor tube. In order to study the effect of reactor tube diameter. TMPTA monomer has been used with ammonium persulfate initiator. The cylindrical tubes with tube size of $6 \mathrm{~mm} \times 125 \mathrm{~mm} .8 \mathrm{~mm} \times 125 \mathrm{~mm}$. $16 \mathrm{~mm} \times$ $125 \mathrm{~mm}$, and $20 \mathrm{~mm} \times 125 \mathrm{~mm}$ were used for the comparative study. For the tubes with $6 \mathrm{~mm}$ and $8 \mathrm{~mm}$ diameter, the silicon caps were used instead of screw cap. The experimental results are summarized in Table 2. Increasing tube diameter. a shift to higher spin modes was observed when the monomer-to-diluent ratio is kept constant, and the Zeldovich number is constant too. The experimental result that the sy'stem moves to higher spin modes by increasing the diameter of the round tube is consistent to the theoretical prediction by Ilyashenko et al. on the universal map of thermal front instabilities. ${ }^{13}$ However, thermal front did not propagate when tube diameter decreased into under a limit size. In this study using TMPTA in DMSO with ammonium persulfate initiator. it was difficult to obtain the propagation of thermal front when the tube diameter was less than $6 \mathrm{~mm}$. Only when we increase the volume percentage of TMPTA in

Table 2. The effect of cylindrical tube diameter on the behavior of the propagating front in the TMPTA frontal polymerization

\begin{tabular}{ccccc}
\hline Volume percentage of & \multicolumn{4}{c}{ Tube diameter (mun) } \\
\cline { 2 - 5 } TMPTA in DMSO $(\%)$ & 6 & 8 & 16 & 20 \\
\hline 28 & n10 propagation & single head & single head & single head \\
32 & no propagation & single head & two heads & two or more heads \\
35 & single head & single head & zig-zag & rippled front \\
40 & single head & single head & flat front & flat front \\
\hline
\end{tabular}




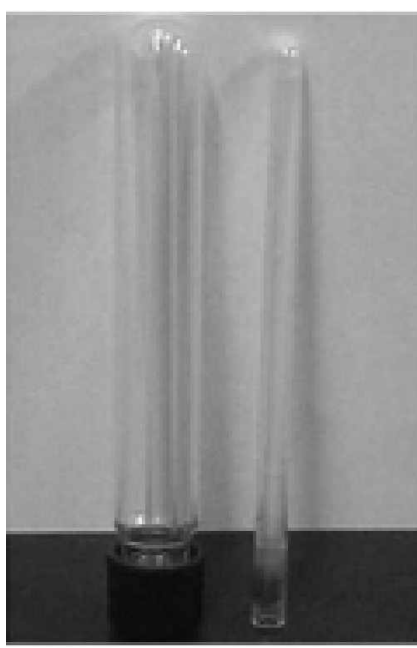

(a)

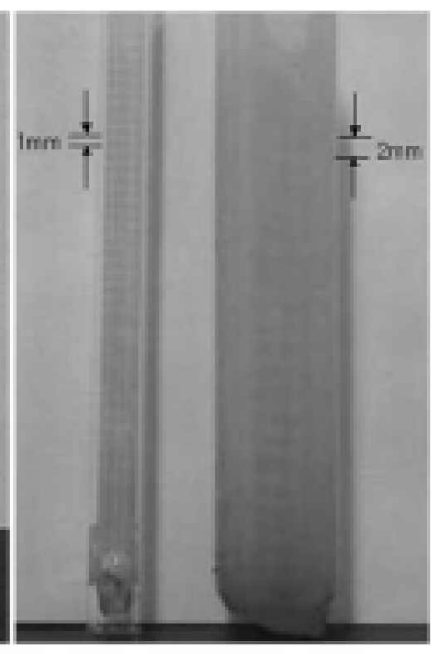

[b]
Figure 5. (a) An experinental scheme for the two-tube system. (b) A typical polymer rod obtained from the two-tube experiments. In the experiment, a $6 \mathrm{~mm} \times 124 \mathrm{~mm}$ round tube is injected into the 16 nun $\times 125 \mathrm{~mm}$ capped tube.

DMSO the thermal front propagated very slowly in $6 \mathrm{~nm}$ tube. It could be interpreted by the heat loss effect of the cylindrical tube reactor. If the diameter of the cylindrical tube decreases more and more. the ratio of surface area to volume of the tube increases gradually. Then, the heat produced in the propagation step of polymerization reaction can be lost more rapidly into the surrounding by the greater surface area of the glass tube. By the result. an autocatalysis reaction step for frontal polymerization can be slowed down. It means that the propagating thermal front is affected much by the heat loss effect in frontal polymerization process.

The heat loss effect by an experiment using two-tubes system. In order to study the heat loss effect of frontal polymerization in the tube with a small diameter in some detail. we have done another experiment by adopting a new experimental systent. In order to protect the heat loss in the tube with a smaller diameter. the frontal polymerization has been performed in a two-tube system in which the tube with a smaller diameter is injected into the tube with a larger diameter as an inner tube. For the experiment. a $6 \mathrm{~mm} \times 124$ $\mathrm{mm}$ round tube was injected into the $16 \mathrm{~mm} \times 125 \mathrm{~mm}$ capped tube. The polymerization was ignited at same time by heating the outer tube by a soldering iron. The same reaction solution was filled up both the inner and the outer tube. In this experiment, the frontal polymerization was always possible in the tube with $6 \mathrm{~mm}$ diameter regardless of the volume percentage of the monomer in DMSO diluent. And the propagating velocity of the inner tube was always same with that of the outer tube. We were not able to follow the behavior of propagating front progressing in the inner tube because of the screen effect by the outer tube. However, the difference of the spin mode of the propagating front in the two tubes was obtained indirectly by the spiral trace imprinted on the polymer rod when the glass tube was broken after the polymerization reaction. A clear spiral trace was also imprinted on the polymer rod obtained in the inner tube and the pitch between spiral trace was some shorter than the trace obtained in the outer tube. The shorter pitch of the spiral trace in the polymer rod obtained from the inmer tube is due to a smaller diameter of the inner tube in spite of same propagating velocity. An experimental scheme for the two-tube system has been introduced in Figure 5(a) and a typical polymer rod obtained from the experiments is shown in Figure 5(b).

\section{Conclusions}

This study shows the reaction condition dependency of the propagating front in frontal polymerization reaction. We have observed some nonplanar spin modes of propagating front including a single-head and two-heads spin mode in diacrylate and triacrylate monomer systems. In some reaction condition. a more complex pattern of a ziz-zag mode was obtained in a triacrylate monomer system. The experimental results that the mode of propagating front in frontal polymerization is very susceptible to the reaction condition and the used monomer were obtained in this study. The effect of tube diameter on the behavior of propagating front has also been studied by a direct and an indirect experimental method. The experimental system using two tubes in a polymerization process has been tried for the indirect method. The experimental result shows that the propagating thermal front is affected nuch by heat loss effect in the frontal polymerization.

Acknowledgment. This paper is dedicated to Prof. K.-H. Jung on the occasion of his retirement. And this work was supported by a grant No. R02-2000-00063 from the Korea Science and Engineering Foundation and BK 21 project of Korean Ministry of Education.

\section{References}

1. Merzhanov. A. G.: Borovinstiava, I. P. Dokl. Nawh SSSR 1972. 204.336.

2. Varma. A.: Lebrat. J. P. Chem. Eng Sci 1992. +7. 2179.

3. Chekanov. Y. A.: Pojmant. T. A. J. Appl Polnt Sei. 2000.78. 2398.

4. Kim. C. Teng. H.; Tucker. C. L.; White. S. R. I. Comp. Hater: 1995. $29,1222$.

5. Dvoryankin, A. V.: Strunina, A. G.; Merzhanov. A. G. Combust. Explos. Shock Waves 1982.18. 134.

6. Sivashinsky. G. I. J. Appt. Math. 1981. 40. 432.

7. Struniti. D. V.: Strutina. A. G.: Rumanov. E. N.: Merzhanov. A. G. Phus. Letts $41994.192,134$.

8. Shkadinsky, K. G.: Khaikin. B. I.: Merzhanov, A. G. Combust. Explos. Shock Haves $1971,1.15$

9. Dattyant. S. P.: Zhirkov. P. V.: Vol'tson. S. A. Russ. Chem. Rev: $198+.53 .150$

10. Pojman. T. A.: Ilyashenko. V. M.: Khan. A. M. J. Chen. Soc. Faraday Trans. $1996,92,2825$.

11. Huh. D. S.; Kim. M. S.; Choe, S. J. Bull. Korean Chem. Soc, 2001. 22. 769 .

12. Pojmant. I. A.: Ilyashentio. V. M.: Khan. A. M. Phrsica D 1995. $8+.260$.

13. Ilyashenko. V. M.: Pojmant. T. A. Chaos 1998. 8.285.

14. Masere. J.: Pojman, J. A. J. Chem Soc. Faraday Trans 1998, 94. 919.

15. Odian. G. Principles of Polymerization: Wiley: New York. 1990. 